\title{
A benchmarking framework for methods to design flexible business processes
}

\author{
Feriel Daoudi ${ }^{1}$ \\ Feriel.Daoudi@malix.univ-paris1.fr \\ ${ }^{1}$ Centre de Recherche en Informatique \\ University Paris 1-Panthéon-Sorbonne \\ 90, rue de Tolbiac \\ 75634 Paris cedex 13 France
}

\author{
Selmin Nurcan ${ }^{1,2}$ \\ nurcan@univ-paris1.fr \\ ${ }^{2}$ IAE de Paris \\ Sorbonne Graduate Business School \\ University Paris 1-Panthéon-Sorbonne \\ 21, rue Broca 75005 Paris France
}

Keywords: flexibility, business processes, methods’ benchmarking, investment, time

\begin{abstract}
The assumption made in this paper is that flexible processes require specific design methods. The choice of a method for modelling flexible processes depends on many criteria and situations that we gathered in a benchmarking framework. The user can use it as a decision support tool to choose the appropriate method in order to design flexible business processes in a given project situation. This framework also includes managerial concerns such as the time and the budget of the project. We use three enterprise modelling techniques to illustrate how to use the proposed framework.
\end{abstract}

\section{Introduction}

The flexibility of business processes depends on the way they are modelled and managed. Indeed the monitoring of business processes can be facilitated by the use of adequate modelling techniques. The best way, from our point of view, is to cover the modelling of those processes from the organisational objectives they allow to be attained, to the software components which support them (in a top-down, bottom-up or mixed way). This way of thinking is partially adopted by software publishers providing panoply of tools to analyse and model enterprises in a global and coherent way. It also underlies Enterprise Application Integration (EAI) solutions that aim to integrate applications in order to avoid discontinuity in business processes they support.

Today, many enterprises tend to adopt a "knowledge management" perspective and store their process models in process warehouses in order to use them as a means to monitor their business processes and to relate them to their organisational structures and perspectives. It seems obvious that some evolution of processes can be simulated on the enterprise models stored in those business process warehouses. To stimulate organisations to use the knowledge management perspective to face the changes that they undergo, managers must be convinced about the benefits of this choice. In fact this requirement represents a strong investment in human and financial resources. Actually stakeholders often doubt the usefulness of business process modelling. Two factors impact the decision of stakeholders/managers. The first one is the time spent on the project before getting the first satisfying result. The second one represents the financial investment which is required compared to the expected benefits. Whatever the purpose of business process modelling is (to handle organizational change or to capitalize on enterprise knowledge), the selection of the appropriate modelling technique is of prime importance. The candidate method should meet project requirements, facilitate the description of business processes which are evolving, and also take into account the managerial factors. Actually, several modelling techniques exist and the selection of the one best fitting those requirements is not easy. On the other hand, benchmarking should not be restricted to just software products but extended to other enterprise modelling approaches. A method-based approach for 
enterprise modelling is a factor impacting both the success of a project as well as the choice of the appropriate software products. Moreover, a modelling method should take into account the managerial factors cited above (time and budget). The difference between technical, business and managerial considerations makes communication between various actors involved in an enterprise project problematic. Indeed managers are the commanders of the vessel, and if the project staff cannot convince them with managerial arguments, the project will never be launched. On the other hand, Information Technology (IT) staff cannot progress if business staff have difficulties with the business process modelling method. Furthermore, benchmarking should not be limited to technical aspects but also include business and managerial views.

In this paper, we propose a benchmarking framework that allows us to evaluate the capabilities of methods to facilitate the design of changing business processes. In addition to various technical factors, this framework also includes two managerial factors: time and budget. Section II introduces the context of work. Section III presents the benchmarking framework and its characteristics (technical factors). Section IV shows the dependencies between these characteristics and the two managerial factors dealt with in this work. Section V illustrates the application of the framework using three modelling environments, EKD-CMM, UML and ARIS. Section VI presents related work. Section VII concludes and presents some research perspectives for our future work.

\section{Context of the work: Evolution of enterprise needs}

Over the two last decades, rapid market changes such as deregulation, globalisation and increased competition have led to a business environment that is constantly evolving. The evolution of Information and Communication Technologies (ICT) and the search for innovative management strategies that could take advantage of them have been pushing organisations into a very competitive and changing environment. Companies change to better satisfy customer requirements, address increasingly tough competition, improve internal processes and adapt the products and services they offer. They have a common business driver: "the urgency of adapting business to the dynamical environment demands". Their capacity to react quickly is often due to their capacity to handle information and communication technologies in support of business evolution requirements.

The process centred view of organisation management was introduced by $\mathrm{M}$. Hammer and $\mathrm{J}$. Champy [8]. During the last decade, business process modelling has gained importance in both the management community and the system engineering community. In fact, in order to take business through a well managed change process, the organisation needs to strike a balance between managerial and technical considerations. This means that a consolidation of the diversity of perspectives that stakeholders, managers, and information system engineers have about the business and the way organisation must change is needed. Thus, while information systems are becoming an integrated part of organisations, the efficient communication between the stakeholders/managers and information system engineers becomes more and more critical because systems should be continuously adapted to changing business practices and needs. Through a better understanding obtained by an explicit representation of their processes, the former aims to improve organisation performance via process reengineering, whereas the latter aims to design/redesign the technological solution that best fits the reengineered processes. Thus, ICT and management go hand in hand in the way of reacting to, adapting and implanting new ways of doing business in today's evolving environments.

In order to respond to these requirements, many process modelling notations have been developed [1], [2], [4], [6], [7], [9], [14], [15], [16], [24]. The market also proposes tools to support definition and management of business processes over time - e.g. ARIS [25], Workflow analyser, Process Model, and Extend+BPR - and ultimately to monitor their enactment with workflow systems [17], [18], [20], [27].

Today companies need (i) to integrate their new applications with their legacy systems and (ii) to orchestrate the execution of their activities and the use of the supporting technological solutions in an integrated environment. This can be achieved by developing process-centric Enterprise Application Integration (EAI) solutions [5], [27]. Process-oriented business management requires an engineering 
environment and appropriate concepts for the design and organization of the business processes and their supporting information systems. The aim is to design and control the organizational structures in a very flexible way so they can rapidly adapt to changing conditions.

\section{Suggested framework for benchmarking methods}

Process flexibility can be perceived in different ways, each determining how to handle it. For us, process flexibility is the capability of the enterprise to react quickly to internal and external changes, and the capabilities offered to users and/or designers to modify business process models and to set up the new enterprise way of functioning. Flexibility is also reflected by the ability of the information system to take into account those changes. This perception of process flexibility highlights the need to have a structured framework to explore the characteristics of candidate methods. Benchmarking should include the considerations of all project actors. In fact, not all process modelling techniques are adequate to satisfy flexibility requirements. The method that has values for the benchmarking criteria that best fit the flexibility definition will be considered as the most appropriate. To specify those characteristics it seems essential to understand the structure of a method.

In the information systems literature, a design method is generally viewed as a 2-tuple: a way of working (Process Model) and a way of modelling (Product Model) [10], [22]. This definition of a method was also applied to business process design and development purposes [13]. The product is a set of diagrams or schema describing the new system to be constructed and the organization in which it will operate [23], [26], [21]. The process describes the road map followed to construct the product. A process and its related product are specific to an application, to a domain or to the whole enterprise. The Product Model defines the set of concepts, their properties and the relationships that are needed to express the outcome of the process. A Process Model is a description of processes at the type level. It defines how to use the concepts defined within a Product Model.

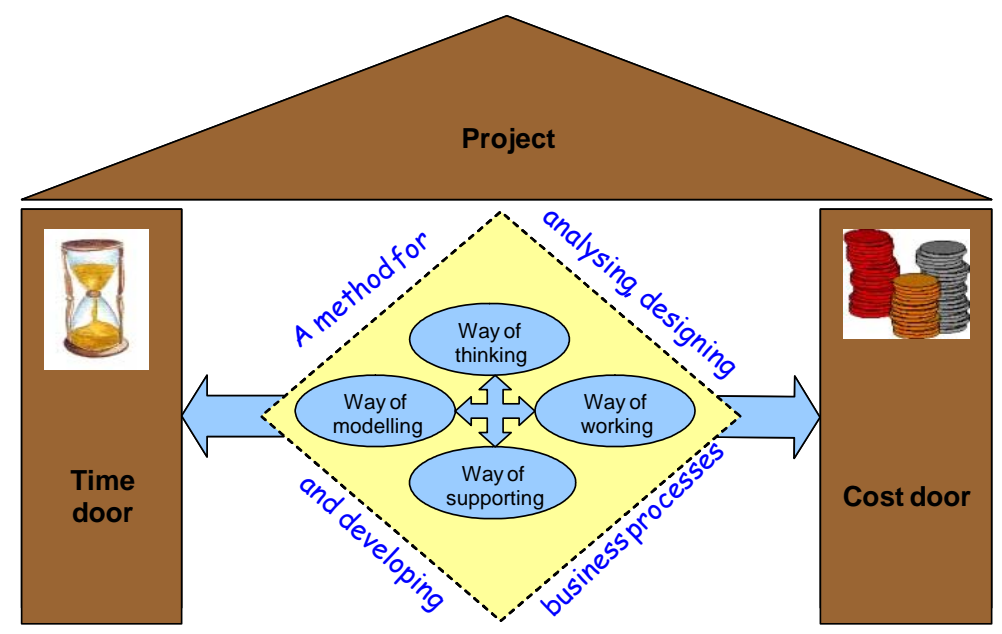

Figure 1. Various perspectives of a business process design and development method

In this paper, the framework proposed is the one depicted in Figure 1. It is inspired by Seligman's framework for the description of information system development methods presented in [26]. As shown in Figure 1, a business process design and development method is described according to four different perspectives:

- The way of thinking verbalizes the assumptions and the philosophy of the benchmarked method to handle flexibility, actors/users, solutions and designers.

- The way of working structures the way in which business process models are analysed and designed. It defines the possible tasks to be performed during the design and development 
process. It provides heuristics on how these tasks should be performed.

- The way of modelling provides information on the modelling concepts, on their properties and on their relationships. It provides a formalism to express business process models.

- The way of supporting refers to software tools that support the design and development of business process models and offers a repository to store and to exploit them. To be efficient, a method gains by being supported by a software tool because the results can be exploited daily and can be produced rapidly.

Each perspective will be detailed using various criteria to handle flexibility as defined above. Actually this definition reflects stakeholders' concerns. Business and IT staff need a method that allows swift translation into diagrams of the enterprise's way of functioning. The enterprise representations (diagrams) aim to improve communication between stakeholders. On the other hand, managers perceive flexibility as a way to adapt the functioning of their enterprise using appropriate resources (budget, time). When a manager talks about flexibility, he/she says "I wish to design new business processes cheaply and quickly". These considerations lead us to propose a set of criteria aimed to improve communication between stakeholders. In fact, the values of those criteria will impact the resources required to obtain this flexibility, respectively time and budget. The combination of these considerations (improving communication, reducing time and budget ...) allowed us to identify ten criteria. Their definition in section III will be followed by a discussion on their impact on managerial factors in section IV.

The proposed set of criteria is a minimal set to examine the ability of a modelling technique to represent flexible business processes. It can be extended if users consider that enterprise flexibility requirements are not reflected enough by those criteria. The specific flexibility requirements can be related to the enterprise activity, its administrative laws, geographical and/or cultural constraints, etc. Because our framework is derived from our definition of flexibility, the addition of situational factors will probably require extending the proposed set of criteria. Although management considerations are evoked through time and budget factors, other situational management factors such as enterprise type, project size, social skills of stakeholders, will not be discussed in this work.

\section{1. Way of thinking}

- Completeness: This criterion describes the various perspectives covered by a method. Obviously, any modelling technique offers its own modelling views. Their number and variety can determine the adoption of the method for a given project. Five perspectives were proposed in [28] that we extend with a sixth: the intentional perspective. The functional perspective characterizes the activities that have to be performed during a business process. The process perspective characterizes the conditions to execute a process. The organization perspective reflects information about the organizational structure and actors to which the business process is intended. The information perspective covers data handled by business processes. The operation perspective describes elementary operations performed by resources and applications. Finally, the intentional perspective represents goals and strategies that the enterprise implements in its business processes. A modelling approach does not necessarily offer concepts to cover the six perspectives. For instance, IDEF0 covers the functional perspective and touches lightly on the information perspective [16]; IDEF1 covers the information perspective and IDEF3 completes them with the process perspective. To model flexible business processes, a method has to provide a minimum toolkit to represent the enterprise elements that are potentially impacted by organizational changes. Without a minimum set of perspectives, the resulting enterprise diagrams will have some deficiencies to highlight all important characteristics of business processes; this can lead to a misalignment between the new business processes and the supporting information system.

- Targeted users: Usually a method is intended for specific kinds of users. As far as business process design is concerned, a method must be intended simultaneously for IT engineers and business actors. Flexibility is ensured when the enterprise can rapidly modify its processes and the 
information system supporting them. Process flexibility involves also the strategic alignment between a business process and its supporting systems. To handle the alignment requirement, business and IT staff should work together to design the new way of working of the enterprise and its new way of supporting. Therefore, the appropriate method should address the two kinds of users and offer autonomy to each team to design the diagrams related to their concerns. It should also facilitate communication, sharing and validation of the resulting enterprise diagrams. For instance, Petri nets address IT staff mastering formal notions whereas BPMN seems to better address business and IT staffs.

\section{2. Way of working}

- Freedom of choice: The aim of this criterion is to ensure that the design activity is not too time and/or resource consuming. In this context, freedom of choice of steps to take helps to minimise the consumption of resources such as time during the design activity. Each method offers a number of steps scheduled in a given way. The degree of modeling freedom offered to the designer related to the execution of these steps varies. More freedom of choice over steps to take allows possible parallelism of design activities and thus time reduction. This means also that some of the proposed modelling formalisms can be used and some others can be omitted according to the situation, leading thus to a resource reduction. This shows how the ad-hoc setting could be advantageous for fast reactivity (this consideration is more applicable in medium/small teams in which each member has a high level of expertise in his/her domain). However, too much freedom of choice over steps to take may slow down the results or can lead to anarchy. It is essential to find the right balance between modelling freedom and framing. Example: (i) in ARIS, users are completely free to start modelling with business objects or functions (ii) afterwards, the central position of the 'function' concept allows designers to visualise artefacts linked to it and to select those which are more essential for the actual modelling purpose (for instance, it is necessary to represent the organizational perspective and actors if the organisational impact of the project is important).

- Guidance: During the application of a method, the ideal is to know which activities should be performed and to obtain guidelines for their selection and execution. Various design methods offer only textual explanations on the underlying modelling formalisms and the enterprise diagrams they allow to be constructed. However, guidance concerning the way of working is seldom provided. In fact, to design enterprise diagrams "easily", and especially flexible business processes, the stakeholders need to be assisted. Guidance should offer orientations relative to the nature of changes (internal, external...) that occur, and the project characteristics (size, budget, risk factors, etc). This ensures the success of the new way of functioning by adopting the adequate modelling perspective that fits the project characteristics. Guidelines can refer also to the appropriate representation perspectives that designers and other stakeholders should adopt in order to ease the implementation of the supporting IS and the application of the new way of functioning. The aim is (i) to assist designers who have to produce enterprise diagrams to integrate the required representation perspectives, (ii) to permit collaboration between stakeholders, and (iii) to facilitate the design of the information system aligned with the business processes previously specified. Guidelines should offer clear and argued choices according to project constraints and design purposes. The aim is to decrease designers' work/costs and to increase the productivity of the project team. Example: EKDCMM provides guidelines to project staff in order to define business models, system specifications, and the relationship between them.

\section{3. Way of modelling}

- Notation: This criterion is related to the expression of enterprise knowledge using representation formalisms. Notation can be formal, semi formal or informal. For providing flexible business process models, this criterion is considered important for two main reasons. First, enterprise diagrams can be drawn more homogeneously when concepts and ways of connecting them are well defined. Second, the notation allows designers to express needed concepts more easily by offering a 
vocabulary and a grammar to describe them. It is also easier to detect inconsistencies on a clearly and rigorously (vocabulary and grammar of the underlying formalism) drawn diagram than in a long textual specification based on natural language. Inconsistencies and lacking concepts are generally more difficult to detect in textual specifications.

- Simplicity: Simple business process models are easier to produce, to understand and to update when change occurs. Simplicity can be characterized by (i) a moderate number of concepts underlying the representation formalism (ii) very significant graphical symbols. For instance IDEF0 provides limited concepts to represent inputs and outputs of a system (functional perspective) without detailing its internal behaviour. Producing business process models with those artefacts is a way for a stakeholder to express (his or her understanding of) the way of functioning of the enterprise, and to share this knowledge with all concerned actors. Simplicity also enables one to introduce changes, to remove diagram chunks, and to detect inconsistencies using readable diagrams, thus accelerating the design of the evolving business process models. Simplicity is often in opposition to richness, and only the project context enables project staff to determine which criteria to emphasise in any given case.

- Richness: Any representation formalism includes the set of concepts intended to represent the enterprise knowledge. The richness lies on the number of those concepts and their power of expression. A formalism is considered rich if it also offers possibilities of extension, as is the case with stereotypes in UML. Despite the improvement in modelling techniques in order to offer a powerful language to represent the enterprise context (allowing the integration of all perspectives), some concepts can still be absent in specific situations. For instance, even if a resource symbol exists in the EPC formalism, a banking company can need a specific symbol to distinguish real time software from other IT products. Thus it seems better to use an extensible formalism to ensure flexibility. The richness permits the integration of specific concepts of the enterprise and especially those related to its environment by adding them, if they are not available in the original Product Model. Finally, as said previously, richness should be controlled because too many concepts may also constitute a brake on straightforward comprehension of enterprise diagrams by all stakeholders.

- Granularity: Granularity represents the level of decomposition and structuring of the enterprise representations. All aspects of the enterprise are affected by this principle. Granularity can be assessed through the number of diagrams required to provide a complete vision of the business. The levels of decomposition of an enterprise diagram can also be determined by it. For instance, an abstract diagram can be progressively detailed into more operational ones according to modelling needs. We argue that granularity affects process flexibility because it facilitates or hinders the understanding of the readers. In fact, abstract diagrams are the means to get a rapid and general idea of the enterprise functioning. Detailed diagrams allow the representation of all crucial details of the enterprise and help managers to take decisions and to introduce wishes and changes. An excessive usage of decomposition results often in voluminous diagrams which are (i) time consuming to design and/or to update and (ii) hard, or at least time consuming, to understand because of the necessity to navigate between sub-diagrams in order to recompose the global enterprise picture. Thus designers should make enterprise diagrams clear and readable for the first level of abstraction, and for all subsequent levels. Furthermore, each stakeholder will deal with the concepts and abstraction levels related to his/her business objectives and participate effectively in the corresponding modelling decisions. For instance, diagrams describing activities as black boxes and their coordination are interesting for managers to determine if their business processes are optimised or aligned with enterprise objectives, while diagrams detailing the underlying operations are interesting for operational managers which have to restructure their organizational unit.

\section{4. Way of supporting}

- Tool support: We argue that a method for designing and developing business processes and 
associated underlying information systems is more powerful if it is supported by a software tool. The level of this support may vary from generic drawing and diagram editors to integrated tool suites that offer guidance to designers through the development process. Handling business processes which are frequently evolving with classical editing tools is time and resource consuming; it is more suitable to exploit integrated tools to obtain high quality process diagrams without time overruns. In changing environments, designers need process warehouses associated with simulation tools in order to anticipate the transformation of enterprise activities and to evaluate the effects of the designed solutions before their implementation. For instance, the MEGA tool allows the simulation of time and human resources required by a business process by providing to designers the capability to specify those resources for each activity. Finally, these tools are often used as learning supports during training sessions that are essential to improve the reactivity of the enterprise when change occurs.

- Connectivity: Business activities are supported by software components developed as parts of the enterprise information system. Those software components should match the requirements of the business process models. For instance, EAI technologies tend to ensure tight communication between them. Through a communication platform, the aim is to keep the transversal integration from the design until the implementation. In other words, the aim is to ensure integration between business domains in organisational and conceptual levels, and between software components in the technical level. This criterion ensures that a method offers mechanisms to cover engineering activities from business process modelling to information system development. Let us remind ourselves that by promoting the enterprise-wide integration, the paradigm of Business Process Management contrasts with traditional information system development; for a long time the latter highlighted the vertical division of the enterprise functions and the construction of information and process islands. Connectivity also permits the reuse (for those components which have proven their efficiency) and the (re)orchestration of existing software components and supports, and thus the evolution of business processes. To attempt connectivity nowadays, people have to invest in compatible tools/formats to develop business scenarios and to implement processes with respect to them.

\section{The link between the benchmarking framework and the managerial factors}

Budget/investment factor: Financial investment in a business process (re)engineering project includes IT and human (knowledge/consulting) investments. The IT investment concerns the software tools that are necessary to design the business processes and to translate them into IT components. The way of supporting view matches completely the definition of the IT investment. The criteria tool support and connectivity represent respectively the design stage of the business processes and their translation into IT components. The budget related to (or impacted by the value of) those criteria is represented by the tool and technical resources of the project.

The human investment is also taken into account by the framework. The criteria that influence directly the human investment are targeted users, guidance and simplicity. Those criteria were chosen because they have a strong link with the methodological and the business knowledge that stakeholders should involve in the design activity. Trainings and consultancies are sometimes more expensive than the software itself. Thus to evaluate the human investment we need to know (i) if the candidate method offers guidance and (ii) if the project staff will need external help. Guidance also allows organizing internal training sessions instead of budgeting expensive external assistance or training (when the solution is provided first or when organisational change occurs and maintenance is required). The simplicity of a method and its targeted users help also to determine if it is better to ask assistance of consultants during the project or to invest in training sessions. For instance, if a method is not well adapted to the profile of the project staff and if, at the same time, it lacks simplicity, it will probably be cheaper to hire a consultant to do the job than to invest in long and expensive training sessions whose results are not guaranteed. On the other hand, if a method targets well the profile of the project staff, 
managers can decide to invest in training sessions and introduce a 'new knowledge' in their organization in order to reproduce it in future projects.

Time factor: For the project manager, the most appropriate method is the one that allows the rapid production of high quality diagrams and also satisfies project specific requirements. The time factor is impacted by the following criteria:

- Completeness: The number of perspectives dealt with causes the time spent during the design stage to vary. To model the whole picture of the enterprise requires more time than if only one perspective has to be analysed and specified. The time spent is, in fact, not only due to the number of perspectives but also to notation, simplicity, richness, and granularity provided by the covered perspectives. Those criteria influence the ability of designers (i) to deal with various modelling techniques and to produce a synthesised and coherent enterprise model, and (ii) to communicate with other stakeholders using the diagrams they have produced. On the other hand, the diversity of perspectives and their capacity to cover the project scope can decrease the time spent for verifying the exactness and consistency of the diagrams, allow traceability between them, and, furthermore, avoid time overruns during the development stage.

- Targeted users: If a method addresses just one type of user, those users should have both functional and technical skills. Usually IT staff who are deeply involved in the requirements analysis in an enterprise and who have a long working experience, have functional and technical skills. In fact, if technical staff do not have the functional knowledge (and this could be considered as the normal way things happen) and if the applied method is specific to them, they will spend more time to find the required business knowledge, to take decisions and to produce IT solutions. In addition, people handle more naturally the modelling concepts close to their expertise domain. It is also possible to extend the knowledge of stakeholders of the selected method by holding training sessions.

- Freedom of choice: When designers have the possibility to focus on steps that they consider as essential based on their own experience, time spent on modelling can decrease. Freedom of choice over steps to take allows us to model those "essential" diagrams in order (i) to respect time constraints and (ii) to delay or cancel some steps during the application of the method. For instance, ARIS designers can model EPC diagrams to (re)design the purchase process and update the function tree diagram only when the process model will be validated by managers.

- Guidance: On the other hand, project staff need methodological guidelines for each specific project situation to avoid the generalisation of the ad-hoc (non repeatable) modelling. Such guidelines allow organization members to improve their modelling knowledge and to rapidly identify best practices such as realising collaborative design sessions, producing deliverables for each major step of the method, and validating those deliverables by the concerned stakeholders. Consultancy services - especially for the methodological aspects - can be considered as less essential for the project if the applied method provides guidelines.

- Notation: A clear notation impacts positively the time factor because it allows us to design business processes in a smooth way without using tricky or complicated means to express the essential concepts of the enterprise. This reduces the time spent for producing and reading diagrams because actors do not need to spend time to check the exactness of the syntax used in them. When the notation is closer to mental models, the following activities can be performed more quickly: designers sketching diagrams, readers understanding diagrams, designers explaining diagrams to project staff, and involved enterprise actors exploiting diagrams.

- Simplicity: Stakeholders can detect more rapidly modelling errors and potential dysfunctions of business processes using diagrams that they can understand without a huge effort, than when using complicated or voluminous models or descriptions (graphical, textual, ...). Thus, decisions to improve processes, role allocations and so on can be better argued and the resulting adaptations or change to the enterprise models can be more rapidly introduced. Moreover, a simple approach also has the advantage that it can be learnt easily, and without external assistance, because the underlying formalism includes neither numerous design rules nor complicated concepts; in this 
way, the time necessary for actors to acquire the ability to apply the approach is potentially reduced.

- Richness: A rich library of symbols attempts to fit better business knowledge and notions to be represented and concepts provided by the formalism which is used. As described earlier, richness is represented also by the capability to add new symbols. This possibility allows project staff to represent naturally some perspectives using specific new symbols, whereas this operation could be more time consuming if only existing - but non appropriate - symbols can be used.

- Granularity: When designers represent the enterprise way of functioning through multiple levels of decomposition, time is also spent in decomposing the global picture into the appropriate levels of representation. It is also necessary to check those diagrams in order to detect the coherence and the traceability between decomposition levels. For instance, when a function is decomposed into sub-functions, it is necessary to verify if the output of the last sub-function in level $n+1$ is similar to the output of the function in the initial diagram (level $n$ ). The impact of granularity on the time factor can be controlled by determining the adequate degree of granularity for the enterprise representation.

- Tool support: Design and development activities are performed faster using a tool supported methodology; the reuse of the business or IS models produced in this environment is also facilitated. Automatic mechanisms facilitate the implementation of the resulting models and allow the exploration of the relationships between various enterprise diagrams. For instance, integrated tools such as ARIS or Case Wise offers navigation possibilities between process diagrams using simple clicks on symbols. Storing enterprise diagrams into process warehouses allows stakeholders to handle and to update them more rapidly than with simple editing tools such as Word or Power Point. In fact, in integrated tools, the modification of an element in a diagram is automatically propagated to all diagrams where this element appears. Simulation possibilities offered by those tools may also decrease the time spent on the design activity; the project staff can thus choose more rapidly between alternative enterprise diagrams.

- Connectivity: Connectivity decreases the time dedicated to the modelling of business processes because it allows for translating them straightforwardly into technical diagrams that can be exploited to implement the corresponding IT components. Connectivity can also impact positively the time factor by supporting the reverse engineering of process models starting from existing IS components. For instance, ARIS for SAPnetweaver allows exploiting business processes implemented in SAP components. The process models extracted from existing IS components can then be improved or corrected to propose a new way of functioning.

\section{Illustration of the benchmarking process}

The benchmarking framework presented above was applied to three business modelling techniques: EKD-CMM [3], ARIS [25] and UML [7]. This study was realised to choose an enterprise modelling approach for a French e-commerce company that redesigned its business processes and the IS supporting them.

Describing the characteristics of the candidate modelling approaches with respect to the benchmarking criteria is the first step of the evaluation process. This step is performed by a method expert. It aims to verify if the project's staff technical requirements will be supported by those approaches. Table 1 shows the result of this step and describes how the studied approaches tackle business process modelling. This table provides the technical description of each technique according to the benchmarking criteria. The values associated with each criterion will help IT and business staff involved in a project to compare their requirements with the possibilities offered by the candidate approaches. The comparison will be done with respect to the reference values shown in the last column of Table 1 . Those reference values are defined beforehand, by the project staff, based on the enterprise context and the flexibility requirements; they express the requirements that the selected approach should satisfy. 
When the project manager examined the values of Table 1, he considered EKD-CMM interesting because it offers guidance to select the adequate strategies corresponding to the situation that project staff is faced with. Nevertheless, the lack of the tool support was a disqualifying point because the main requirement of the business team was to have a tool for designing and storing the produced diagrams, making simulations and also using them for learning and knowledge capitalization purposes - in other words to sustain the ability to change and improve. 


\begin{tabular}{|c|c|c|c|c|}
\hline $\begin{array}{l}\text { Criteria/ } \\
\text { Methods }\end{array}$ & EKD-CMM & ARIS & UML & $\begin{array}{l}\text { Reference } \\
\text { values }\end{array}$ \\
\hline Completeness & $\begin{array}{l}\text { process }+ \text { informational+ } \\
\text { intentional + functional } \\
+ \text { operational } \\
+ \text { organisational }\end{array}$ & $\begin{array}{l}\text { process+informational + } \\
\text { intentional }+ \\
\text { functional+operational+ } \\
\text { organisational }\end{array}$ & $\begin{array}{l}\text { process +informational } \\
\text { +functional } \\
\text { +operational } \\
\text { +organisational }\end{array}$ & $\begin{array}{l}\text { functional }+ \\
\text { operational }+ \\
\text { informational }\end{array}$ \\
\hline Targeted users & Business staff, IT staff & Business staff, IT staff & Business staff, IT staff & $\begin{array}{c}\text { Business and IT } \\
\text { staff }\end{array}$ \\
\hline Arbitrariness & Moderate & Moderate & Moderate & High \\
\hline Guidance & Supported by maps & No & No & Yes \\
\hline Notation & Semi formal & Semi formal & Semi formal & Semi formal \\
\hline Simplicity & High & $\begin{array}{l}\text { Moderate (many } \\
\text { concepts and connectors } \\
\text { difficult to use) }\end{array}$ & $\begin{array}{l}\text { Moderate (many } \\
\text { concepts difficult to } \\
\text { use) }\end{array}$ & High \\
\hline Richness & $\begin{array}{l}\text { Moderate, adding new } \\
\text { concepts is not possible }\end{array}$ & $\begin{array}{l}\text { High, adding new } \\
\text { concepts is not possible }\end{array}$ & $\begin{array}{l}\text { High, adding new } \\
\text { concepts is possible } \\
\text { with stereotypes }\end{array}$ & High \\
\hline Granularity & Multiple levels & Multiple levels & Multiple levels & Not essential \\
\hline Tool & No & ARIS HOBE & MEGA & Yes \\
\hline Connectivity & No & SAP R/3, \#EAI & \#EAI & Yes \\
\hline
\end{tabular}

Table 1. Comparison between ARIS, UML and EKD-CMM using the proposed framework

This comparison was followed by the evaluation of those approaches with respect to time and budget factors (Table 2 and Table 3). The scoring uses_the sign $(\uparrow)$ when the value of the criterion for the corresponding approach (in Table 1) has a potential negative impact on (increases) the evaluated factor, time or budget. The sign $(\downarrow)$ will be used when the value of the criterion for the corresponding approach (in Table 1) has a potential positive impact on (decreases) the evaluated factor. The evaluation is done with respect to the reference values established beforehand (last column of Table $1)$.

\begin{tabular}{|c|c|c|c|}
\hline $\begin{array}{ll} & \text { Methods/ } \\
\text { Criteria } & \end{array}$ & ARIS & UML & EKD-CMM \\
\hline Completeness & $\uparrow$ & $\downarrow$ & $\uparrow$ \\
\hline Targeted users & $\downarrow$ & $\downarrow$ & $\downarrow$ \\
\hline Arbitrariness & $\downarrow$ & $\downarrow$ & $\downarrow$ \\
\hline Guidance & $\uparrow$ & $\uparrow$ & $\downarrow$ \\
\hline Notation & $\downarrow$ & $\downarrow$ & $\downarrow$ \\
\hline Simplicity & $\uparrow$ & $\uparrow$ & $\downarrow$ \\
\hline Richness & $\downarrow$ & $\uparrow$ & $\uparrow$ \\
\hline Granularity & $\uparrow$ & $\uparrow$ & $\uparrow$ \\
\hline Tool & $\downarrow$ & $\downarrow$ & $\uparrow$ \\
\hline Connectivity & $\downarrow$ & $\downarrow$ & $\uparrow$ \\
\hline Score of $(\downarrow)$ & 6 & 6 & 5 \\
\hline
\end{tabular}

Table 2. Time factor

\begin{tabular}{|c|c|c|}
\hline $\begin{array}{ll}\text { Criteria } & \text { Methods/ }\end{array}$ & ARIS & UML \\
\hline Targeted users & $\downarrow$ & $\downarrow$ \\
\hline Guidance & $\uparrow$ & $\uparrow$ \\
\hline Simplicity & $\downarrow$ & $\uparrow$ \\
\hline Tool & $\downarrow$ & $\uparrow$ \\
\hline Connectivity & $\downarrow$ & $\downarrow$ \\
\hline Score of $(\downarrow)$ & 4 & 2 \\
\hline
\end{tabular}

Table 3. Budget factor

\footnotetext{
Legend:

- The sign $(\downarrow)$ means that the value of the criterion corresponding to this line (defined in Table 1) has the potential to decrease the time (or

budget) spent for the method corresponding to this column.

- The sign $(\uparrow)$ means that the value of the criterion corresponding to this line (defined in Table 1) has the potential to increase the time (or budget) spent for the method corresponding to this column.

- The cell 'score of' corresponds to the number of $(\downarrow)$ signs
}

If a cell value in Table 2 or 3 , describing the position of an approach with respect to a criterion, borders on the reference value given for this criterion, then the potential impact of this technical criterion on the evaluated managerial factor (time or budget) is positive, in other words the time or the budget will decrease. Otherwise time/budget can potentially increase. The aim of the scoring process is to calculate the number of $(\downarrow)$ signs. The approach having the highest number of $(\downarrow)$ is the one impacting more positively the evaluated factor. The effect on the project will be to control the risk of 
time/cost overruns due to the use of inappropriate methods. Other risk factors which should also be examined are out of the scope of this paper.

Table 2 shows the result of the evaluation with respect to the time factor. For instance, for the completeness criterion, UML has a $(\downarrow)$ sign, whereas ARIS and EKD have each a $(\uparrow)$ sign. The best score is associated with the approach that provides the minimal set of perspectives including the required ones (reference value of completeness). Nevertheless, the necessity to integrate correctly the various perspectives in order to reflect exactly the global vision of the enterprise was neglected in this project. As a matter of fact, the capabilities provided by the studied approach to integrate diagrams of different perspectives should also be taken into account when the impact on the time factor is analysed. Table 2 shows also that EKD-CMM obtained the worst score (smallest number of $(\downarrow)$ )). This is especially due to the lack of the tool support and the connectivity. ARIS and UML achieved the same score.

The evaluation with respect to the budget factor was performed for the two approaches, ARIS and UML. Table 3 shows the result. MEGA (a process modelling tool widely used in France) was chosen as a tool support for UML because it fills also the requirements of other already planned projects in this enterprise. MEGA also allows connectivity through a software component that can be connected to EAI platforms and provide them input and output parameters of the activities of a business process. The evaluation of the impact of the tool support on the budget factor was made particularly regarding the acquisition cost of those tools; ARIS Webdesigner is cheaper than MEGA. With respect to guidance, simplicity and targeted users criteria, the cost for the internal staff training for ARIS Webdesigner will be cheaper than a training package offered by the publisher of MEGA. Finally, ARIS was chosen by the project manager, after discussion with all stakeholders, expecting that it will allow designers to model business processes more quickly and cheaply than UML with MEGA.

\section{Related work}

In the business process modelling literature, few benchmarking frameworks exist. The first review of methods, techniques and tools used in business process reengineering was presented in [11]. This empirical review was completed using a reference framework called step-activity to categorize tools and techniques useful for IT projects and all their dimensions (strategy, people, management, structure, technology) [11]. The step-activity framework decomposes the lifecycle of a project into different stages (envision, initiate, diagnose, redesign, reconstruct, evaluate) and enumerates existing techniques and associated tools dedicated to the activities that compose each stage. This categorization highlights neither the characteristics of those techniques nor the project situational factors. Nevertheless, this work inspired other work, for instance [12] that proposed a framework composed of three dimensions to classify existing process modelling techniques. The horizontal dimension represents the business goals for which the modelling activity is performed (understanding, process improvement, process management, process development, process execution). The vertical dimension represents the various perspectives that a given technique provides. The last one, named the fit dimension, represents the kind of projects for which such modelling techniques can be applied. This framework is descriptive; its main advantage is the classification of existing techniques according to the three cited dimensions. It does not include the situational factors of the project in order to select the adequate technique. It does not explore either the characteristics of the studied modelling techniques in order to determine their adequacy with the actual project situation. In addition to those classification works, the Q-ME framework [13] aims to assess the quality of process modelling approaches or techniques. The authors present a set of quality properties (expressiveness, arbitrariness, etc) that they link to the blocks of their meta-model (way of modelling and way of working). This framework, based on a set of evaluation criteria, allows a deeper analysis than the descriptive ones adopted in [11] and [12]. Nevertheless, this evaluation does neither include the enterprise context nor link with the user profile/knowledge. 


\section{Conclusion}

The continuous economical and technological evolution pushes enterprises to adapt frequently their way of functioning. The flexibility requirement has stressed multiple works in managerial, organizational and IT areas in order to facilitate the implementation of these changes. The work presented in this paper concerns the benchmarking of modelling techniques/approaches with respect to their capacity to design flexible business processes. We argue that the choice of a process modelling technique should not be ad-hoc. Benchmarking techniques need to be extended to process modelling approaches because the latter represent an essential factor for the success of projects dealing with the design and implementation of business process support systems.

In this paper, a benchmarking framework was presented in order to assist project staff to select a modelling technique which offers the capacity to design flexible business processes. This framework is derived from a flexibility definition reflecting the usual concerns of IT projects stakeholders. Three main purposes appear in this definition: facilitate communication between stakeholders and avoid time and cost overruns. The framework is composed of four perspectives: way of thinking, way of modelling, way of working and way of supporting.

This work was validated by a case study where a project staff benchmarked ARIS, EKD-CMM and UML in order to select the most appropriate process modelling approach for an e-commerce company. The framework helped the project staff to express clearly their requirements concerning process modelling techniques. It also permitted them to deal with two essential managerial factors: time and budget.

The proposed benchmarking framework is context centred. It is based on a generic definition of flexibility through a set of technical criteria and two managerial factors: time and budget. The benchmarking framework constitutes a brainstorming tool to highlight both the advantages and the missing features of candidate methods according to the usual project management concerns. It is a generic framework in which other evaluation criteria, reflecting more specific flexibility requirements of a given enterprise related to its business processes, can be added.

The proposed set of benchmarking criteria is minimal and can be extended according to the enterprise perception of flexibility that varies according to situations that enterprises encounter. $\mathrm{We}$ intend to examine them exhaustively in our future works. The integration of enterprise factors is crucial for an optimal use of the proposed framework. Project factors (time, budget, management support, size, etc), organizational factors (organization structure, size, vision, etc), human factors (technical knowledge, social skills, etc) are an outline of the situational factors listed in [19]. To integrate those situational factors, we need to apply our framework to other enterprises undergoing such change processes in order to determine the benchmarking criteria that must be added accordingly. This future empirical study will also permit us to complete the situational factors listed in [19].

\section{References}

[1] P. Armenise, S. Bandinelli, C. Ghezzi and A. Morzenti, A survey and assessment of software process representation formalisms. Int. Journal of Software Engineering and Knowledge Engineering, 3(3), 1993.

[2] S. Bandinelli, A. Fugetta and S. Grigoli, Process Modelling in the large with SLANG. Proceedings of the $2^{\text {nd }}$ Int. Conference on Software Process, Berlin, Germany, 1993, p. 75-93.

[3] J. Barrios and S. Nurcan, Model Driven Architectures for Enterprise Information Systems, Proceedings of the CAISE'04 International Conference, Springer Verlag (pub), 2004.

[4] J. Bubenko, Enterprise Modeling. Ingénierie des Systèmes d'Information, Vol 2, N 6, 1994.

[5] R. T. Burlton, Business Process Management- Profiting from process, SAMS Publishing, 2001.

[6] S. Decker, M. Daniel, M. Erdmann and R. Studer, An enterprise reference scheme for integrating Model based knowledge engineering and enterprise modeling. $10^{\text {th }}$ European Workshop on Knowledge 
Acquisition, Modeling and Management, EKAW'97, Lecture Notes in Artificial Intelligence, SpringerVerlag, Heidelberg, 1997.

[7] H.-E. Eriksson and M. Penker, Business modeling with UML-Business patterns at work, J. Wiley, 2000.

[8] M. Hammer and J. Champy, Re-engineering the Corporation: A Manifesto for Business Revolution. New York: Harper Collins Publishers, inc., 1993.

[9] D. Harel, STATEMATE: A working environment for the development of complex reactive systems. IEEE Transactions on Software Engineering, 16, 4, (April 1990), 403-414.

[10] A.F. Harmsen, S. Brinkkemper and H. Oei, Situational Method Engineering for Information System Projects. In Olle T. W. and A. A. Verrijn Stuart (Eds.), Methods and Associated Tools for the Information Systems Life Cycle, pp. 169-194, Proceedings of the IFIP WG8.1 Working Conference CRIS'94, NorthHolland, Amsterdam, 1994.

[11] W.J. Kettinger, J.T.C. Teng and S. Guha, Business Process Change: A Study of Methodologies, Techniques and Tools, MIS Quartely, 21(1), March, 1997, p. 55-80.

[12] G.M. Giaglis, A taxonomy of business process modelling and information systems modelling techniques. International Journal of Flexible Manufacturing Systems, 13(2):209-228, 2001.

[13] B.J. Hommes and V. Van Reijswoud, Assessing the quality of business process modelling techniques, Proceedings of the $33^{\text {rd }}$ HICSS International Conference, 2000.

[14] I. Jacobson, M. Chisreton, P. Jonsson and G. Overgaard, Object Oriented Software Engineering - A Use Case Driven Approach, Addison-Wesley, 1993.

[15] S. Jarzabek and T.W. Ling, Model-based support for business reengineering. Information and Software Technology, $\mathrm{N}^{\circ} 38$ (1996), 355-374.

[16] D.A. Marca, and C.L. McGowan, IDEF0/SADT: Business Process and Enterprise Modeling. San Diego: Eclectic Solutions, Inc., 1993.

[17] D.R. McCarthy and K. Sarin, Workflow and transactions in InConcert, Bulletin of Technical Committee on Data Engineering, 16(2) IEEE, Special Issue on Workflow and Extended Transactions Systems, 1993.

[18] R. Medina-Mora, T. Winograd, R. Flores and F. Flores, The Action Workflow approach to workflow management technology. Proceedings of the CSCW'92 International Conference, Toronto, Canada, 1992.

[19] S. Nurcan and C. Rolland. A multi-method for defining the organizational change. Journal of Information and Software Technology, Elsevier. 45:2(2003), p. 61-82.

[20] A.M. Palermo and S.C. McReady, Workflow software: A primer, Proceedings of the Conference Groupware'92, London (1992).

[21] M.P. Papazoglou and W.-J. van den Heuvel, Configurable business objects for building evolving enterprise models and applications, Business Process Management, Van de Aast W, Desel J., Oberweis A. (eds), Springer, 2000.

[22] N. Prakash, On Method Statics and Dynamics, Information Systems, 24(8), p. 613-637, 1999.

[23] C. Rolland, P.Loucopoulos, G. Grosz and S. Nurcan, A framework for generic patterns dedicated to the management of change in the electricity supply industry. $9^{\text {th }}$ International DEXA Conference and Workshop on Database and Expert Systems Applications, August 24-28, 1998, p. 907-911.

[24] J. Rumbaugh, M. Blaha and W. Premerlani, F. Eddy, and W. Lorensen, Object Oriented Modeling and Design. Prentice-Hall, 1991.

[25] A.W. Scheer, W. Jost, F. Abolhassan, Business Process Excellence: Aris in Practice, Springer Verlag, 2002.

[26] P.S Seligmann, G.M. Wijers and H.G. Sol, Analyzing the structure of IS methodologies, an alternative approach, Proceedings of the First Dutch Conference on Information Systems, Amersfoort, the Netherlands, 1989.

[27] W.M.P. van der Aalst and K. van Hee, Workflow Managament: models, methods and systems, MIT Press, 2002. 
[28] W.M.P. van der Aalst, A. ter Hofstede, M. Weske (Eds.): Business Process Management. Proceedings of the International Conference on Business Process Management 2003. Springer Lecture Notes in Computer Science 2678. Heidelberg: 2003. 